\title{
Clinicopathological characteristics and prognosis of pulmonary spindle cell carcinoma (PSCC): a population-based retrospective study
}

\author{
Jiangzhou Zhang, Yanli Yan, Beina Hui, Shuheng Bai, Wen Ma, Juan Ren \\ Department of Radiotherapy, Oncology Department, First Affiliated Hospital of Xi'an Jiaotong University, Xi'an, China \\ Contributions: (I) Conception and design: J Ren; (II) Administrative support: J Zhang; (III) Provision of study materials or patients: Y Yan; (IV) \\ Collection and assembly of data: B Hui; (V) Data analysis and interpretation: S Bai, W Ma; (VI) Manuscript writing: All authors; (VII) Final approval \\ of manuscript: All authors. \\ Correspondence to: Juan Ren. Department of Radiotherapy, Oncology Department, First Affiliated Hospital of Xi'an Jiaotong University, Xi'an 710061, \\ China. Email 869491533@qq.com.
}

\begin{abstract}
Backgroundk In consideration of the scarceness and importance of histological analysis, the clinic pathological features of pulmonary spindle cell carcinoma (PSCC) were comprehensively analyzed in the present work to improve the treatment and deepen our understanding of the disease.

Methods: Data of the PSCC patients from 2008 to 2013 in the Surveillance, Epidemiology, and End Results (SEER) database were acquired, analyzed and contrasted to that of the subjects with non-small cell lung cancer (NSCLC). Overall survival (OS) was evaluated based on the Kaplan-Meier method, univariate analysis (UVA) and multivariate analysis (MVA) were applied for the Cox proportional hazards regression. The risk factors related to 1-, 3-, and 5-year OS in PSCC subjects were identified.

Results: The data of 171 subjects considered to suffer from PSCC were collected and compared with that of 41,438 NSCLC patients. There was a poor differentiation in $72.9 \%$ of PSCC, and $44.4 \%$ were at the stage IV of American Joint Committee on Cancer (AJCC). The median OS time of PSCC was 8 months [95\% confidence interval (CI): 6.23-10.72] with 5-year OS as 23.9\% (95\% CI: $21.5-25.7 \%$ ). Tumors of PSCC were significantly undifferentiated, which exhibited the higher rate to be resected by surgery, with more lymph node metastases and distant metastases than that of NSCLC $(\mathrm{P}<0.001)$. It was demonstrated in UVA and MVA that the $\mathrm{N}$ stage, $M$ stage, and surgery served independently as the risk factors of OS. The calibration variable for the nomogram was 0.735 (lower than 0.8 ).

Conclusions: There were specific clinicopathologic features in PSCC. The results revealed that there was an independent correlation between $\mathrm{N}$ stage, $\mathrm{M}$ stage, or surgery with OS. However, 1-, 3- and 5-year OS could not be precisely predicted by the nomogram.
\end{abstract}

Keywords: Lung spindle cell carcinoma; clinicopathology characteristics; survival

Submitted Sep 13, 2020. Accepted for publication Dec 31, 2020.

doi: 10.21037/apm-20-1841

View this article at: http://dx.doi.org/10.21037/apm-20-1841

\section{Introduction}

Pulmonary spindle cell carcinoma (PSCC) is a special malignancy with distinctive clinicopathological features and prognosis. Unique clinical and pathological characteristics and prognosis have seldom been illustrated yet. Even diagnosed at the early stage, PSCC showed an unsatisfied prognostic outcome (1-3). Lung biopsy is crucial for the patients suffered from PSCC, due to the incompletion of preoperative diagnosis under most circumstances. According to the 2015 World Health Organization (WHO) histological classification of lung tumors, pulmonary sarcomatoid carcinoma (PSC) could be divided into 
PSCC, pleomorphic carcinoma (PC), giant cell carcinoma (GCC), carcinosarcoma, and pulmonary blastoma (4). Five cases diagnosed with computed tomography (CT) scans demonstrated that central, low-density regions were observed in the lesions, which might be the most specific imaging feature of PSCC. Kida et al. suggested that the above characteristic was associated with the hemorrhage at the center of lesions (2). With a predominant advantage in detecting metastatic lesions, positron emission tomography/ CT (PET/CT) has been broadly utilized (5). Clinical symptoms and images of PSCC were similar to other lung cancers with a lack of specificity. Belonging to the high malignancy, PSCC rarely performed as a low-grade malignant tumor in histological morphology. Besides, PSCC can be manifested by a variety of morphological and histological characteristics which was easily misdiagnosed. Therefore, efforts should be emphasized on distinguishing PSCC from other tumors during the pathological examination to avoid misdiagnosis. It has been reported that immunohistochemistry and genetic analysis played vital roles in the definitive diagnosis of PSCC (3).

High aggressiveness was considered as the characteristic of PSCC compared with other subtypes of non-small cell lung cancer (NSCLC) (6). Up to now, there have been no large-scale researches focused on PSCC, and clinical characteristics, diagnosis, and treatment strategy of PSCC were still unclearly interpreted. Furthermore, no standard treatments have been developed for PSCC based on chemotherapy, traditional Chinese medicine (TCM), surgery, and radiation therapy. Although the treatments of Chinese medicine or carboplatin combined with albumin paclitaxel had been proven to extend the survival of PSCC in case reports, further studies with larger sample size were still needed $(6,7)$. Although the sensitized mutation of epidermal growth factor receptor (EGFR) was observed in the PSCC, the treatments exhibited no marked effects. Targeted therapy and immunotherapy for the PSCC were only at the initial stage $(8,9)$, the molecular mechanisms of which on PSCC needed to be further investigated. Since the clinical characteristics, diagnostic methods and therapies of PSCC were still superficial, it was of great importance to deeply interpreting the clinicopathologic features and prognosis and confirming the valid therapeutic strategy.

In the present work, clinical and pathological features and long-term prognosis of the subjects with PSCC were analyzed based on the database of a large national population, in order to summarize its clinical characteristics affecting survival compared with other NSCLC. We present the following article in accordance with the STROBE reporting checklist (available at http://dx.doi.org/10.21037/ apm-20-1841).

\section{Methods}

\section{Data extraction}

The information of PSCC patients with the diagnosis and treatment between 2009 and 2013 were acquired from the Surveillance, Epidemiology, and End Results (SEER) database (http://seer.cancer.gov/) based on SEER*Stat software ver 8.3.5 (ver 8.3.5, https://seer.cancer.gov/ seerstat/). The subjects determined by the third edition of International Classification of Diseases for Oncology (ICD-O-3), with the histological code 8032/3, were selected in this cohort study. The inclusion criteria were represented as follows: the subjects with single primary tumor, malignant PSCC verified by histology, complete follow-up information, and basic information such as age and ethnicity. The SEER database was applied to get access to the signed authorization and permission for the application of dataset. The approval was waived by the local ethics committee, due to the public availability and de-identification of the data in SEER. The study was conducted in accordance with the Declaration of Helsinki (as revised in 2013).

\section{Variables in the study}

The following referenced information could be obtained from the SEER data, including age, sex, ethnicity, ICD-0-3 Hist(Histologic type)/behavior, grading of tumors, primary site of tumors, time of diagnosis, tumor size, TNM stage of the primary tumor, historic phase, surgical resection or not, type of operation (e.g., pneumonectomy, lobectomy, bilobectomy and sublobar resection), radiotherapy or not, chemotherapy or not, survival performance, and vital status. The adjustment of TNM stage was artificially performed according to the criteria of the sixth edition of American Joint Committee on Cancer (AJCC). The age at diagnosis was redefined using 10 -year categorical intervals when establishing the nomogram for prediction. The categories of lung operation contained an "other" option.

\section{Survival data}

Overall survival (OS) was defined as the duration from the 
definite diagnosis time until the death time for any causes or the last follow-up time. The survival time of the patients with less than 1 month survival were considered as zero in SEER database. The survival of those patients was supposed to be 0.5 month according to the standard conventions of epidemiology.

\section{Statistical analysis}

The Kaplan-Meier method was adopted to plot the statistical analysis cumulative survival curves for every variable of patients and the log-rank test was applied for the comparison of the results. Cox proportional hazards regression was utilized to identify the indicators with prognostic value, which were shown as hazard ratios (HRs).

The parameters with $\mathrm{P}<0.1$ in univariate analysis (UVA) were also consisted of multivariate analysis (MVA). The nomogram was established based on the data of MVA, which was published in former studies. The predictive error was evaluated using 200 bootstrap samples. The evaluation of model performance was performed using the concordance index (C-index) and calibration plots. The discrimination capability was estimated with $\mathrm{C}$-index ranging from 0.5 to 1.0 . Nomogram plot calibration was adopted to evaluate the overall agreement between the predictive and actual survival, which has been indicated previously. Statistical analyses were conducted based on $\mathrm{R}$ version 3.5.2 software (https://www.rstudio.com/). The R package consisted of survival, rms and ggplot 2 . The twosided $\mathrm{P}<0.05$ was considered as statistically significant.

\section{Results}

\section{Clinical information}

Based on the demography and features of clinical pathology, 167,181 NSCLC patients meeting the standards were totally selected in the SEER database, including 142 cases $(0.10 \%)$ suffered from PSCC.

As listed in Table 1, 57.7\% of the SEER cohort was made up of men, suggesting that there was a faint male predominance in PSCC, namely, men were more vulnerable to PSCC than women. Compared with 48 cases in the lower lobe $(33.8 \%)$ and 11 in the middle lobe (7.7\%), PSCC was preferred to develop in the upper lobe with 74 patients $(52.1 \%)$, indicating that the upper lobe of lung was the most susceptible to PSCC. Histological analysis showed that grades III and IV (poorly differentiated or undifferentiated) form the majority. It indicated that most of the lesions in PSCC were poorly differentiated or undifferentiated. As for the PSCC patients, the later the stages were, the lower the proportions were, which suggested that there were probably still chances of the operation for some PSCC patients.

\section{Pathological characters}

It was revealed that markedly poorly differentiated tumors, the extensive pleural invasion, high probability of radical resection through surgery, $\mathrm{N}+$ disease, and distant metastases were the characteristics of PSCC compared with NSCLC patients $(\mathrm{P}<0.05)$.

\section{Survival data}

The median OS time of the 142 subjects suffered from PSCC in our work was 5 months (95\% CI: 3.14-6.86) with the 5-year OS rate $15.5 \%$ (95\% CI: 9.4-21.6\%). In the NSCLC group, the median OS was 15.2 months (95\% CI: 14.0-16.4) with a 5-year OS percentage of $18.0 \%$ (95\% CI: 17.8-18.2\%). Figure 1 displayed the Kaplan-Meier curves of OS. The median survival and 5-year survival rates of PSCC were lower than those of NSCLC.

To sum up, 142 subjects were dead in the duration of follow-up studies, in which 121 deaths were caused specifically by PSCC and the rest died of other causes, containing heart disease $(10.6 \%)$ and kidney and renal pelvis disease $(4.9 \%)$.

It was indicated in Figure 2 that cumulative survivals of subjects were Shown in different age, sex, and race groups. As revealed in Figure $2 A$, there were no significant differences in the survival probability among subjects with the age <50, 50-59 and 60-69 years. Likewise, the survival rate of patients aged $70-79$ and $\geq 80$ years differentiated insignificantly. OS was dramatically decreased in the subjects aged $\geq 70$ years than in other groups $(\mathrm{P}=0.000)$. For different sex groups, there were no remarkable differences as displayed in Figure $2 B(\mathrm{P}=0.755)$. Similarly, Figure 3 indicated that the OS data was similar among various racial groups $(\mathrm{P}=0.155)$.

For subjects with grade I or II diagnosis and whose tumors could be well or moderately differentiated, they were excluded for there were only 6 such cases. It was demonstrated in Figure $3 A$ that there was no conspicuous difference in OS between the patients at grade III (poorly differentiated) and those of grade IV (undifferentiated) $(\mathrm{P}=0.634)$. The information of pleural invasion in 2010 was 
Table 1 Comparison of the baseline data of PSCC and NSCLC subjects

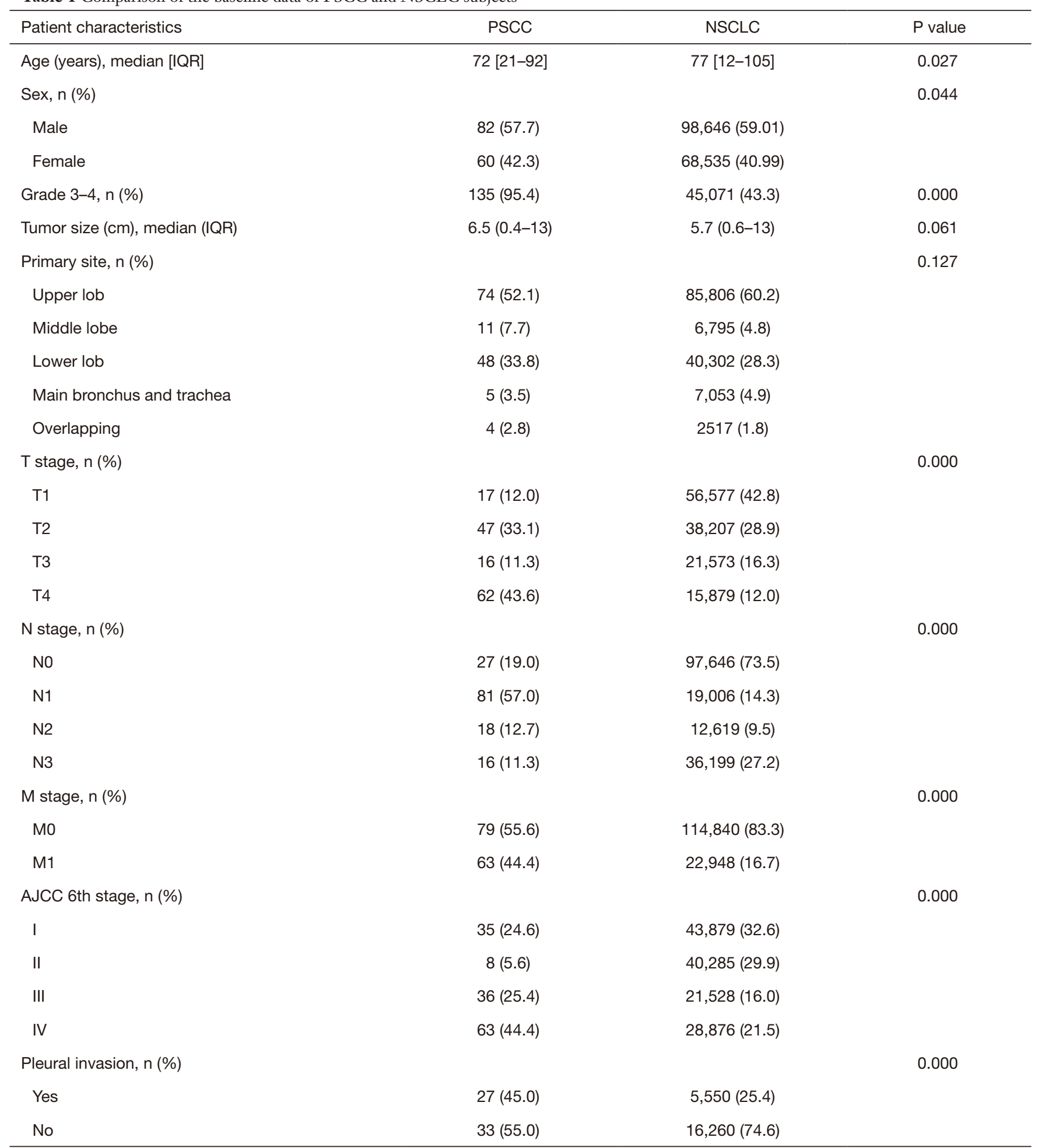

Table 1 (continued) 
Table 1 (continued)

\begin{tabular}{|c|c|c|c|}
\hline Patient characteristics & PSCC & NSCLC & $P$ value \\
\hline Performed & $47(33.1)$ & $38,312(22.9)$ & \\
\hline Not performed & $95(66.9)$ & $128,869(77.1)$ & \\
\hline Surgical method, n (\%) & & & 0.030 \\
\hline Partial/wedge/segmental resection & $11(25.0)$ & 7,602 (23.2) & \\
\hline Pneumonectomy & $4(9.1)$ & $1,547(4.7)$ & \\
\hline Radiation, n (\%) & & & 0.047 \\
\hline Performed & $50(35.2)$ & $46,380(27.7)$ & \\
\hline Performed & $47(33.1)$ & $50,688(30.3)$ & \\
\hline No/unknown & $95(66.9)$ & $116,493(69.7)$ & \\
\hline
\end{tabular}

PSCC, pulmonary spindle cell carcinoma; IQR, interquartile range; OS, overall survival; NSCLC, non-small cell lung cancer.

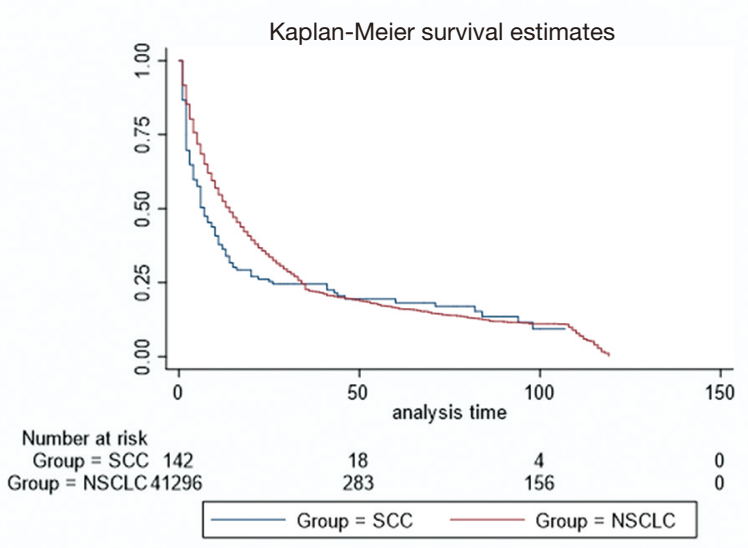

Figure 1 The evaluation of overall survival of pulmonary spindle cell carcinoma (SCC) and NSCLC patients based on Kaplan-Meier. The total survival of NSCLC was better than that of SCC $(\mathrm{P}=0.015)$. NSCLC, non-small cell lung cancer.

obtained, in which 27 subjects (45.0\%) with some cases at the early stage were found. It was illustrated in Figure $3 B$ that the OS of the patients whose tumors extended to the visceral or parietal pleural was dramatically reduced than that of the subjects without invasion $(\mathrm{P}=0.026)$. Additionally, the OS of AJCC stage were markedly distinct as revealed in Figure $3 C(\mathrm{P}=0.000)$. For the subjects at stage $\mathrm{I}$, the median
OS was 60 months (95\% CI: 20.20-99.80) with the 5-year OS rate as $47.6 \%$. There were only three cases in stage II which were not enough for the analyses. Median OS were 7 months (95\% CI: 5.03-8.97) and 2-year OS 13.2\% for stage III, and 2 months (95\% CI: 1.39-2.61) and 2-year OS $5 \%$ for stage IV.

In Figure $4 A$, the OS of the cases undergoing operations was predominantly elevated $(\mathrm{P}=0.000)$. However, radiotherapy $(\mathrm{P}=0.110$, Figure $4 B)$ or chemotherapy $(\mathrm{P}=0.701$, Figure $4 C)$ had no significant effects on OS, which varied from different surgical procedures $(\mathrm{P}=0.000)$. The median OS for the subjects underwent lobectomies was 60 months (95\% CI: 13.54-106.46) vs. 19 months (95\% CI: 11.09-26.91) for partial/wedge/segmental resection vs. 11 months (95\% CI: $0.88-21.12$ ) for pneumonectomy $(\mathrm{P}=0.011)$. There were $27(19.0 \%)$ cases at the $\mathrm{pN} 0$ stage for the nodal status and $115(81.0 \%)$ at the $\mathrm{pN}+$ stage. The median OS of the subjects at the pN0 stage was 11 months (95\% CI: 4.20-17.80) and that at the $\mathrm{pN}+$ patients was 6 months (95\% CI: 4.17-7.83) $(\mathrm{P}=0.000)$.

The covariates with $\mathrm{P}<0.1$ identified in UVA were contained in MVA. Since there was a strong association among the pleural invasion, AJCC stage, and TNM stage, they were excluded in the MVA avoiding the collinearity of variables. Table 2 listed the Cox proportional hazards regression of UVA and MVA. MVA illustrated that $\mathrm{N}$ stage 
A

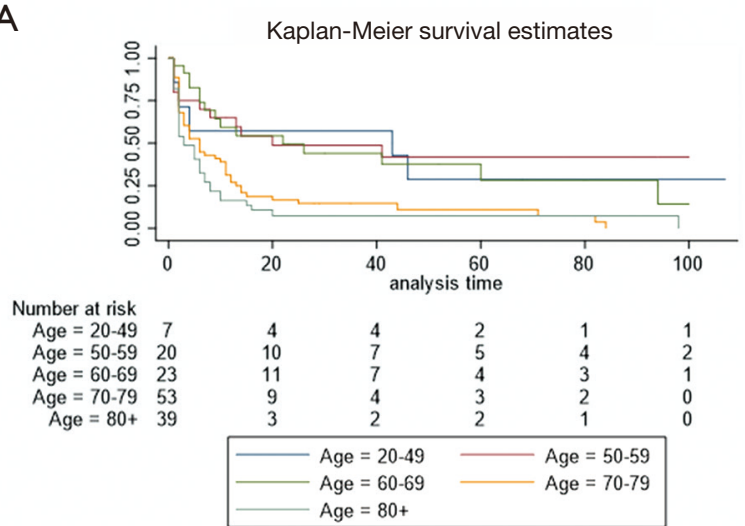

B

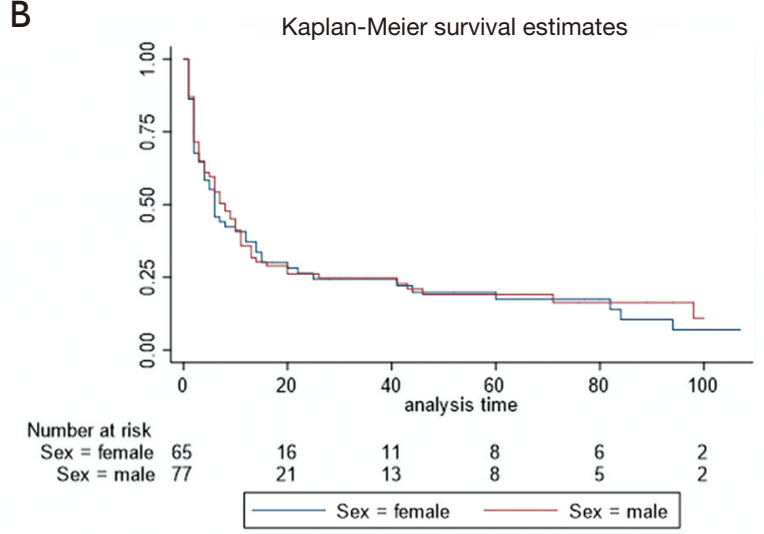

C

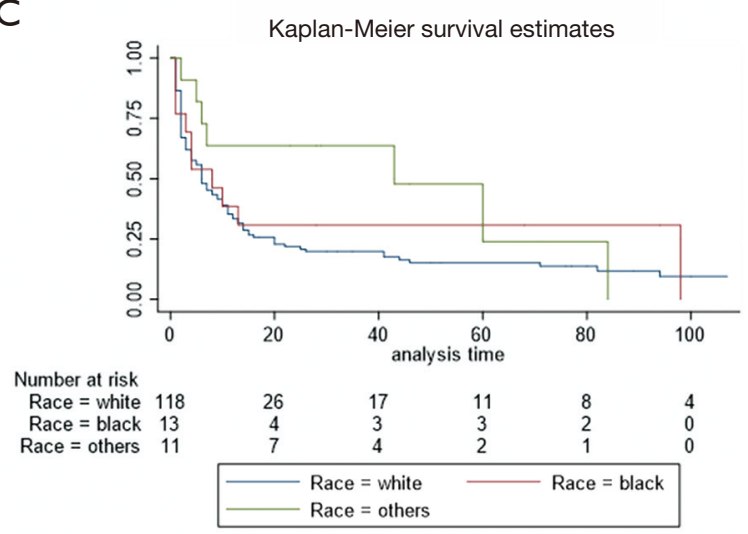

Figure 2 The overall survival was estimated based on Kaplan-Meier analyses by (A) age, (B) sex, (C) race.

( $\mathrm{P}=0.047), \mathrm{M}$ stage $(\mathrm{P}=0.008)$, and surgery $(\mathrm{P}=0.000)$ could be served as independent predictors of survival.

Figure 5 displayed the nomogram consisting of the factors independently associated with survival. The parameters corresponding to the features of subjects were added to evaluate 1-, 3-, or 5-year OS. The c-index of the nomogram
A

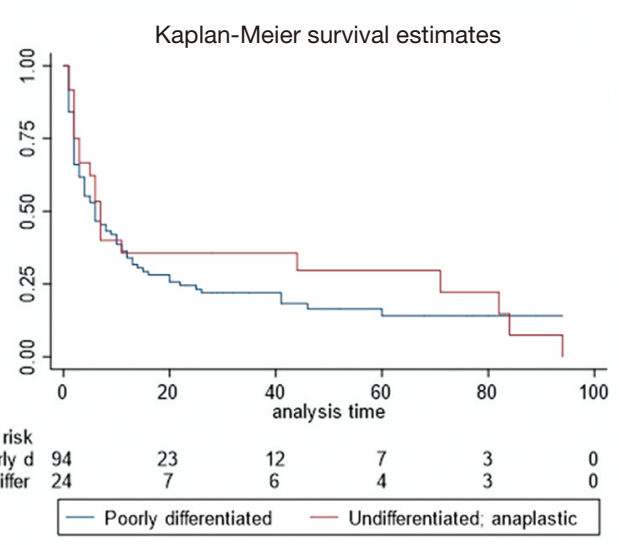

B

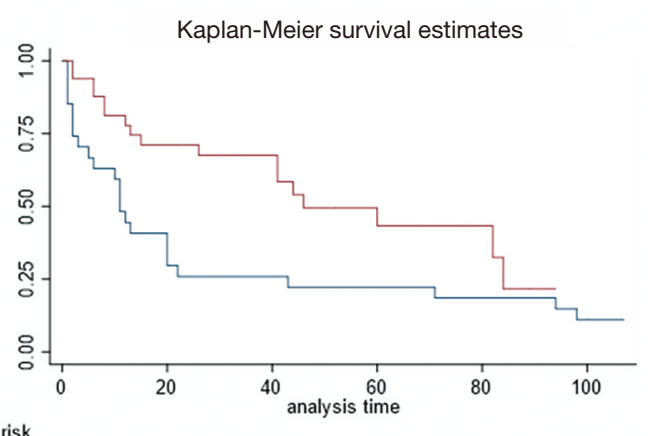

Number at risk

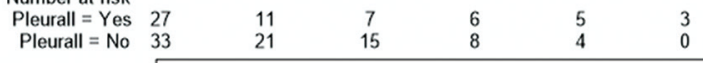

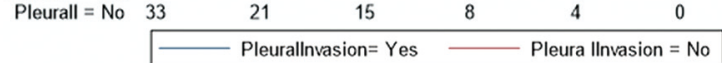

C

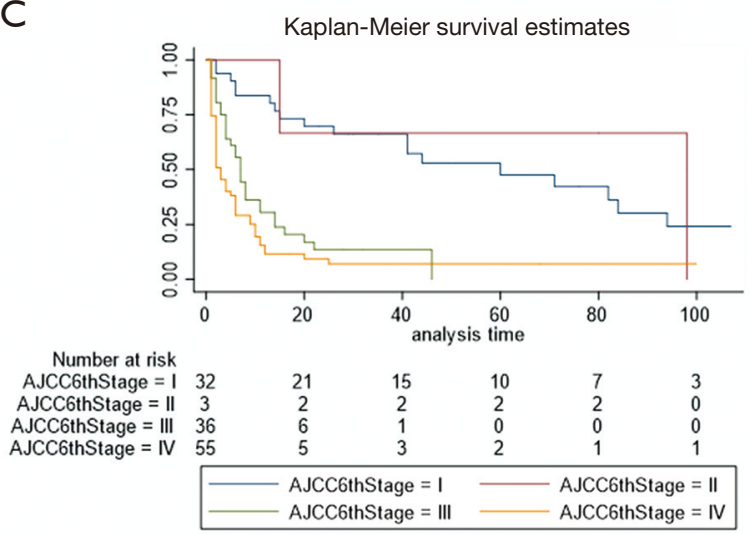

Figure 3 The assessment of overall survival based on Kaplan-Meier analyses by (A) tumor grade, (B) pleural invasion, and (C) AJCC stage. AJCC, American Joint Committee on Cancer.

was 0.735 , not suggesting the discrimination capability. It was revealed in Figure 6 that the calibration of the predictive accuracy based on the nomogram represented that there was a satisfied agreement with the actual and predicted 1-, 3-, and 5-year OS, with a slope approach to 45. 
A

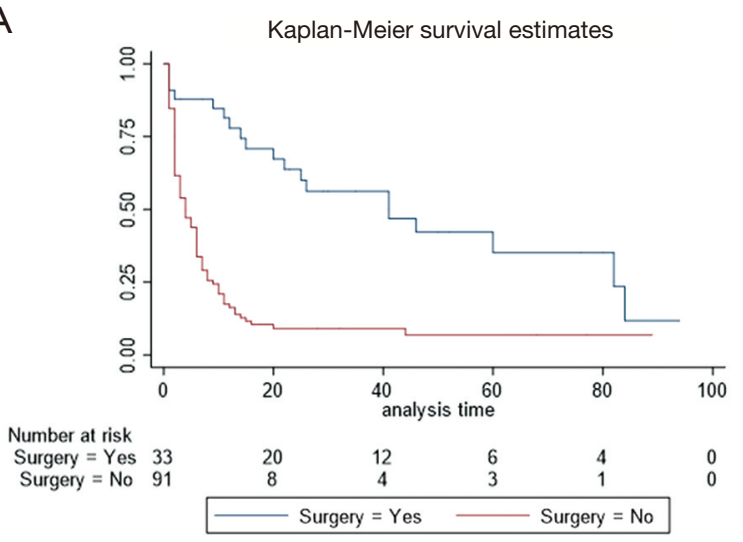

B

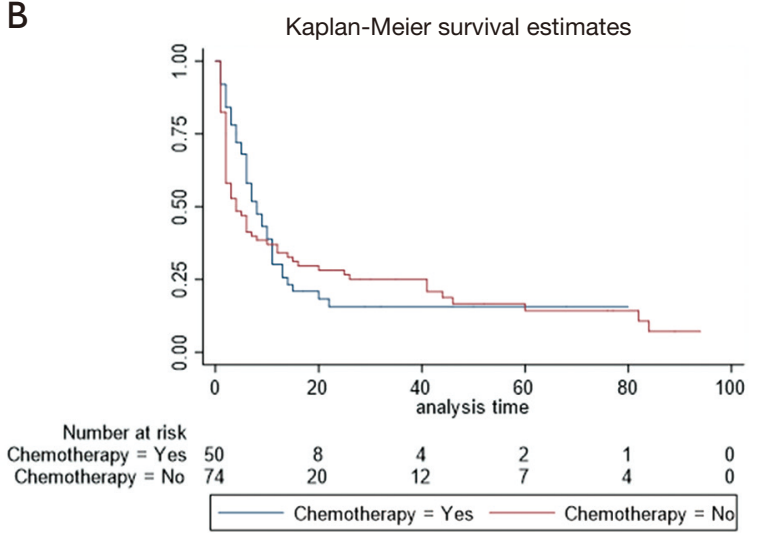

C

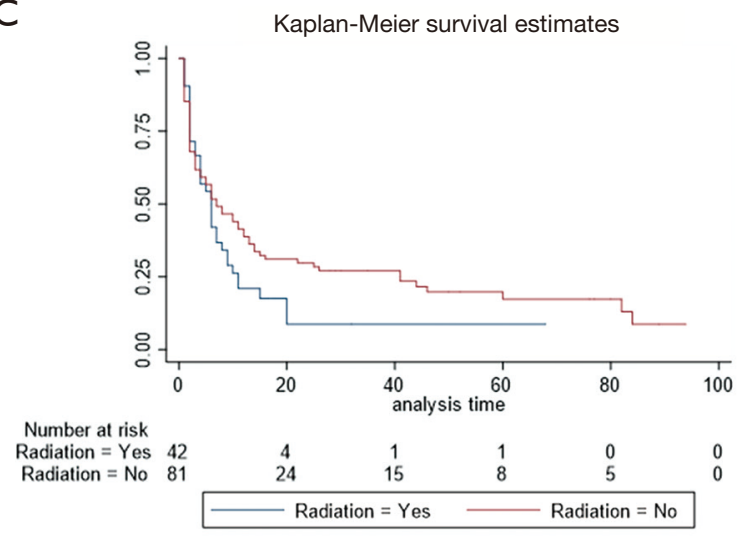

Figure 4 the overall survival of subjects treated by (A) surgery, (B) chemotherapy, or (C) radiotherapy based on Kaplan-Meier analyses.

\section{Discussion}

Since all the types of sarcomatoid carcinoma have a low incidence, PSCC is a very rare form, accounting for only $0.2 \%$ to $0.3 \%$ of all lung cancers (10). The clinical course of PSCC was more aggressive than other malignant lung tumors. Comparing with NSCLC, the pleura or chest wall was often invaded by PSCC, making the operation more difficult. However, these subjects were treated promptly and the prognosis was satisfied, especially for the patients at early stage.

The findings in our study were crucial for the treatment of PSCC. There were 18 out of 142 PSCC patients who survived for more than 50 months, illustrating that early diagnosis and treatment may play vital roles in the extension of the survival.

There were remarkable correlations among $\mathrm{N}$ stage, $M$ stage, AJCC stage, and surgery with survival outcomes based on MVA, and the results of Cox regression were applied to establish the nomogram for predicting 1-, 3-, and 5 -year OS. For the operability, the nomogram would be served as an available tool for physicians and patients, illustrating that surgery played a robust role in OS.

Ikushima et al. reported that gefitinib had no conspicuous effects on the subjects, which indicated that the development of PSCC was not or only partially relied on the EGFR signaling pathway and the pathogenesis of PSCC may mainly be triggered by other mechanisms (8). It was of great importance for further investigating the dominant signaling pathways linked to PSCC, which may promote the evolution of therapeutic strategies against PSCC (11-15). Tsuji et al. (7) applied carboplatin and nanoparticle albuminbound paclitaxel to lengthen the survival of patients. However, further study could be conducted to confirm the regime.

It was indicated by Li et al. that the long-term survival of the subjects suffered from PSCC could be realized by treating with TCM (traditional Chinese medicine). Increasing evidences proved that TCM had the distinct effect on improving the qualities of life and served as the promising drug for extending the survival of patients with tumors (6). Therefore, the development of novel target therapy, chemotherapy, and complementary medicine for patients with cancer was urgently needed; $75 \%$ of the lung sarcomatoid cancers (pleomorphic, spindle cell and giant cell) were positive for PD-L1. The PD-L1 expression of prognosis was unsatisfied, on the other hand, which made the subjects suited for the targeted immunotherapy (3).

Multidisciplinary diagnostic methodology for PSCC played vital roles in its diagnosis and treatment. In view of the insufficient number of cases, further investigations and studies should be carried out to better interpret the clinical characteristics, behaviors and biological features of primary PSCC. 
Table 2 Univariate and multivariate Cox proportional hazards analysis.

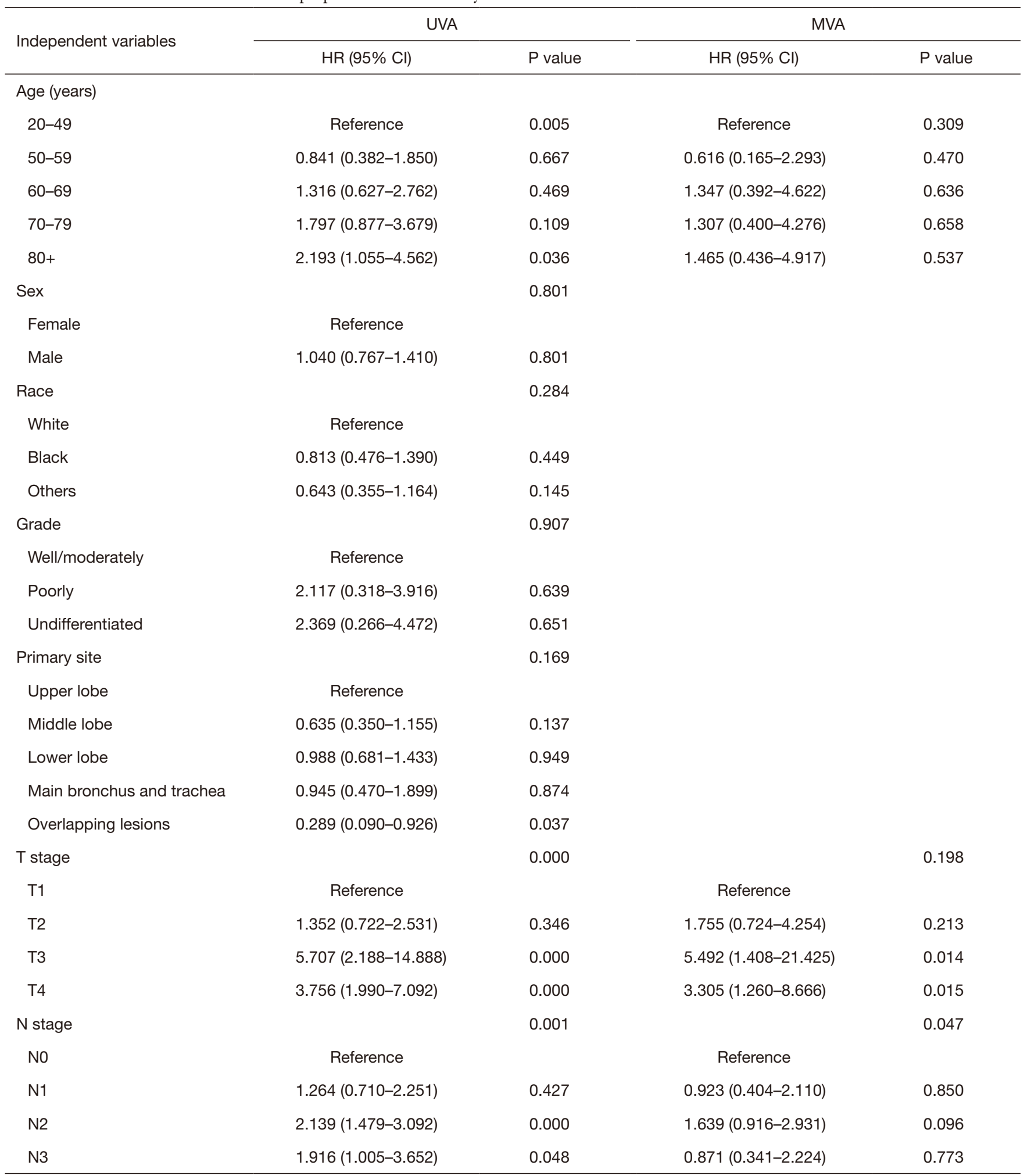

Table 2 (continued) 
Table 2 (continued)

\begin{tabular}{|c|c|c|c|c|}
\hline Independent variables & \multicolumn{2}{|c|}{ UVA } & \multicolumn{2}{|c|}{ MVA } \\
\hline M stage & & 0.000 & & 0.008 \\
\hline MO & Reference & & Reference & \\
\hline M1 & $2.540(1.827-3.531)$ & 0.000 & 2.951 (2.364-3.538) & 0.008 \\
\hline I & Reference & 0.000 & & \\
\hline II & $0.753(0.229-2.474)$ & 0.641 & & \\
\hline III & $2.682(1.609-4.470)$ & 0.000 & & \\
\hline IV & $4.289(2.748-6.696)$ & 0.000 & & \\
\hline Not performed & Reference & & Reference & \\
\hline Performed & $2.993(1.985-4.511)$ & 0.000 & $3.329(1.754-6.320)$ & 0.000 \\
\hline Radiation & & 0.170 & & \\
\hline Not performed & Reference & & & \\
\hline Performed & $0.778(0.544-1.113)$ & 0.170 & & \\
\hline Chemotherapy & & 0.339 & & \\
\hline No/unknown & Reference & & & \\
\hline Performed & $1.182(0.839-1.666)$ & 0.339 & & \\
\hline
\end{tabular}

UVA, univariate analysis; MVA, multivariate analysis; HR, hazard ratio.

Points
Nstage
Mstage
Surgery
Total points
1-year survival
3-year survival
5-year survival

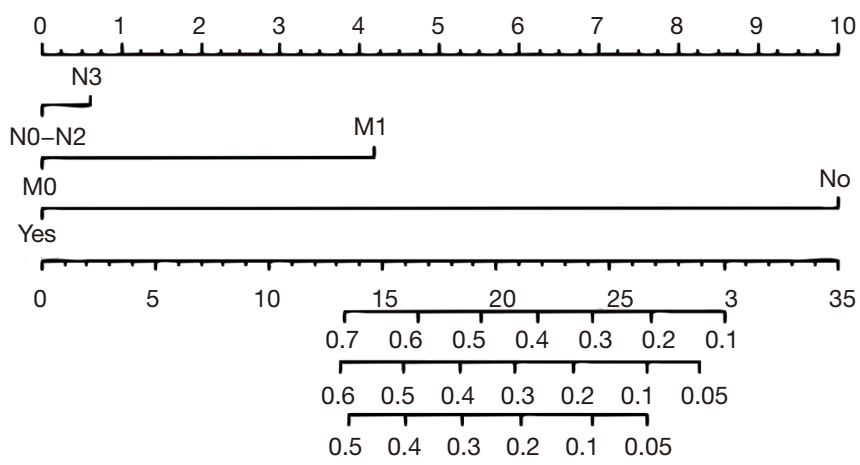

Figure 5 The 1-, 3-, and 5-year overall survival of subjects suffered from spindle cell carcinoma (SCC) based on the nomogram. 


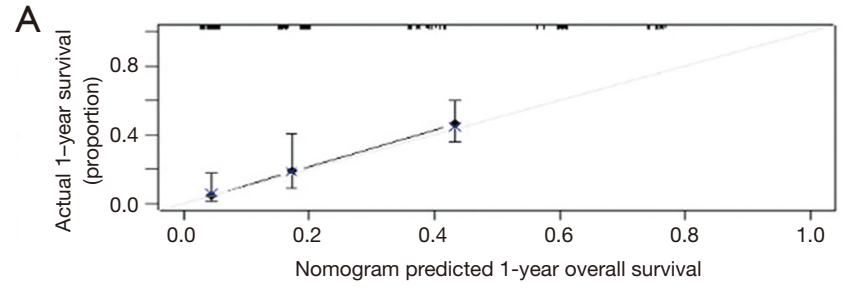

B

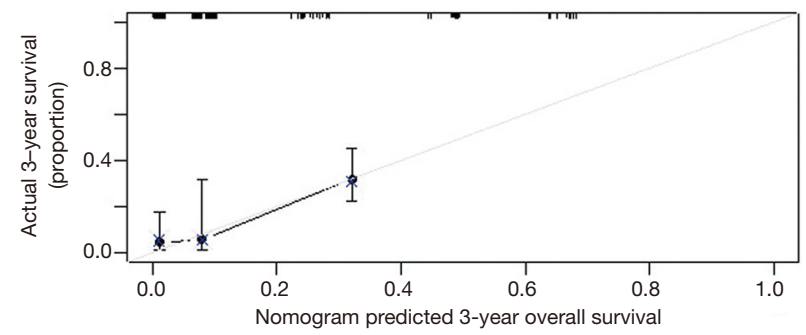

C

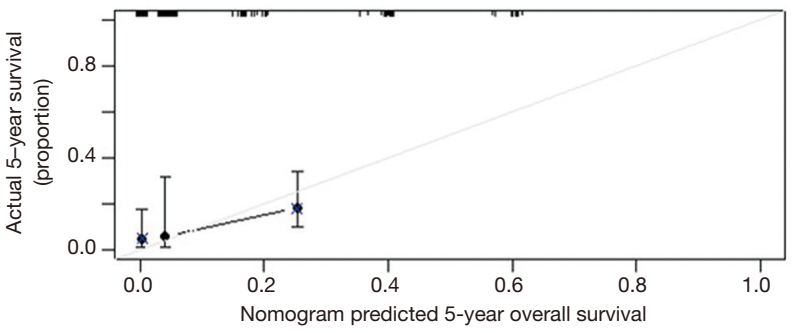

Figure 6 The calibration of the prediction of (A) 1-year, (B) 3-year, and (C) 5-year overall survival of SCC subjects based on the nomogram. The black line revealed the actual probability was agreed with the predicted ones. SCC, spindle cell carcinoma.

\section{Conclusions}

There were specific clinicopathologic features in PSCC. The results revealed that there was an independent correlation between $\mathrm{N}$ stage, $\mathrm{M}$ stage, or surgery with OS. However, 1-, 3- and 5-year OS could not be precisely predicted by the nomogram.

\section{Acknowledgments}

Funding: This manuscript is supported by the National Natural Science Foundations of China (Juan Ren, 81772793/H1621, 31201060/C0709; 30973175/H1621; 81772793/H1621).

\section{Footnote}

Reporting Checklist: The authors have completed the STROBE reporting checklist. Available at http://dx.doi. org/10.21037/apm-20-1841
Conflicts of Interest: All authors have completed the ICMJE uniform disclosure form (available at http://dx.doi. org/10.21037/apm-20-1841). The authors have no conflicts of interest to declare.

Ethical Statement: The authors are accountable for all aspects of the work in ensuring that questions related to the accuracy or integrity of any part of the work are appropriately investigated and resolved. The study was conducted in accordance with the Declaration of Helsinki (as revised in 2013). The approval was waived by the local ethics committee, due to the public availability and deidentification of the data in SEER.

Open Access Statement: This is an Open Access article distributed in accordance with the Creative Commons Attribution-NonCommercial-NoDerivs 4.0 International License (CC BY-NC-ND 4.0), which permits the noncommercial replication and distribution of the article with the strict proviso that no changes or edits are made and the original work is properly cited (including links to both the formal publication through the relevant DOI and the license). See: https://creativecommons.org/licenses/by-nc-nd/4.0/.

\section{References}

1. Feng L, Cai D, Muhetaer A, et al. Spindle cell carcinoma: the general demographics, basic clinico-pathologic characteristics, treatment, outcome and prognostic factors. Oncotarget 2017;8:43228-36.

2. Kida J, Kanaji N, Kishi S, et al. An Autopsy Case of Rapidly Progressing Spindle Cell Carcinoma of the Lung Accompanied with Intratumor Hemorrhage. Am J Case Rep 2015;16:805-10.

3. Qi DJ, Liu B, Feng L, et al. Pulmonary spindle cell carcinoma with unusual morphology: A rare case report and review of the literature. Medicine (Baltimore) 2017;96:e7129.

4. Annede P, Cosset JM, Van Limbergen E, et al. Radiobiology: Foundation and New Insights in Modeling Brachytherapy Effects. Semin Radiat Oncol 2020;30:4-15.

5. Ettinger DS, Wood DE, Aisner DL, et al. Non-Small Cell Lung Cancer, Version 5.2017, NCCN Clinical Practice Guidelines in Oncology. J Natl Compr Canc Netw 2017;15:504-35.

6. Li W, Chen M, Zhao Y. Long-term survival in a patient with pulmonary spindle cell carcinoma treated with traditional Chinese medicine. BMJ Case Rep 
2018;2018:bcr2018225989.

7. Tsuji T, Kim YH, Ozasa H, et al. Successful treatment with carboplatin and nanoparticle albumin-bound paclitaxel in a patient with pulmonary spindle cell carcinoma. Respir Med Case Rep 2015;15:48-50.

8. Ikushima H, Sakatani T, Masuda Y, et al. Lung spindle cell carcinoma harbouring a constitutively active epidermal growth factor receptor mutation. Respirol Case Rep 2018;7:e00395.

9. Yvorel V, Patoir A, Casteillo F, et al. PD-L1 expression in pleomorphic, spindle cell and giant cell carcinoma of the lung is related to TTF-1, p40 expression and might indicate a worse prognosis. PLoS One 2017;12:e0180346.

10. Mainwaring MG, Poor C, Zander DS, et al. Complete remission of pulmonary spindle cell carcinoma after treatment with oral germanium sesquioxide. Chest 2000;117:591-3.

11. Lee CK, Man J, Lord S, et al. Checkpoint Inhibitors in Metastatic EGFR-Mutated Non-Small Cell Lung

Cite this article as: Zhang J, Yan Y, Hui B, Bai S, Ma W, Ren J. Clinicopathological characteristics and prognosis of pulmonary spindle cell carcinoma (PSCC): a population-based retrospective study. Ann Palliat Med 2021;10(4):3793-3803. doi: 10.21037/apm-20-1841
Cancer-A Meta-Analysis. J Thorac Oncol

2017;12:403-7.

12. Oshima Y, Tanimoto T, Yuji K, et al. EGFR-TKIAssociated Interstitial Pneumonitis in Nivolumab-Treated Patients With Non-Small Cell Lung Cancer. JAMA Oncol 2018;4:1112-5.

13. Liu X, Jia Y, Stoopler MB, et al. Next-Generation Sequencing of Pulmonary Sarcomatoid Carcinoma Reveals High Frequency of Actionable MET Gene Mutations. J Clin Oncol 2016;34:794-802.

14. Takakuwa O, Oguri T, Uemura T, et al. Osimertinibinduced interstitial lung disease in a patient with non-small cell lung cancer pretreated with nivolumab: A case report. Mol Clin Oncol 2017;7:383-5.

15. Schrock AB, Li SD, Frampton GM, et al. Pulmonary Sarcomatoid Carcinomas Commonly Harbor Either Potentially Targetable Genomic Alterations or High Tumor Mutational Burden as Observed by Comprehensive Genomic Profiling. J Thorac Oncol 2017;12:932-42. 